\title{
Cost-Effectiveness of Tolvaptan in Autosomal Dominant Polycystic Kidney Disease
}

\author{
James Lineen, MB BCh, MSc and David M.J. Naimark, MD, MSc on behalf of the \\ Sunnybrook Nephrology Journal Club \\ Sunnybrook Health Sciences Centre and Institute of Health Policy, Management, and Evaluation, \\ University of Toronto, Toronto, Ontario, Canada
}

\section{TO THE EDITOR}

Erickson and colleagues (1) have published an elegant Markov-based cost-effectiveness analysis that extends the results of the TEMPO (Tolvaptan Efficacy and Safety in Management of Autosomal Dominant Polycystic Kidney Disease and Its Outcomes) trial (2) of tolvaptan for the treatment of adult autosomal dominant polycystic kidney disease (ADPKD). The authors indicate that a key input parameter was the change in estimated glomerular filtration rate (eGFR) in patients who would or would not receive chronic tolvaptan therapy. In fact, the parameter that was modeled was the change in the actual GFR. However, because the eGFR is a nonlinear function of the GFR, the change in eGFR does not numerically equal the change in GFR.

For example, taking the average starting serum creatinine level and GFR that were observed in the TEMPO trial and assuming that net creatinine production would be constant over 1 year, one can calculate the expected change in serum creatinine level for a given change in GFR and use the Chronic Kidney Disease Epidemiology Collaboration equation (3) to compute the resulting expected change in eGFR. The observed difference in the change in eGFR between the tolvaptan and placebo groups in the TEMPO trial of $0.98 \mathrm{~mL} / \mathrm{min}$ per year corresponds to a difference in the change in GFR of $0.88 \mathrm{~mL} / \mathrm{min}$ per year. The latter discrepancy is small over 1 year. However, when extrapolated over the decades that patients are likely to live with ADPKD, the authors' model would substantially overestimate the beneficial effect of tolvaptan to retard progression to end-stage renal disease.

In cost-effectiveness analyses, a key model outcome is the incremental cost-effectiveness ratio. For the tolvaptan model, where the numerator of the incremental cost-effectiveness ratio (incremental, discounted, lifetime costs) is large and the denominator (incremental, discounted quality-adjusted life-years) is small, a further reduction in the denominator would greatly increase the computed ratio. The result of this consideration is that the authors may have underestimated the incremental cost-effectiveness ratio for tolvaptan therapy for ADPKD. However, this would only bolster their conclusion that, given the current cost of the drug, it is not a cost-effective treatment strategy.

Potential Conflicts of Interest: None disclosed. Forms can be viewed at www.acponline.org/authors/icmje/ ConflictOfInterestForms.do?msNum_L13-1102. 
In general, lack of numerical equivalence between the change in eGFR and the change in GFR is probably of little importance when the change in eGFR is used as a surrogate for end-stage renal disease because the change in eGFR and the change in GFR correlate highly. However, we believe that in clinical trials where the primary outcome of interest is the change in GFR, the use of change in eGFR may lead to an inaccurate estimate of benefit.

\section{References}

1. Erickson KF, Chertow GM, Goldhaber-Fiebert JD. Cost-effectiveness of tolvaptan in autosomal dominant polycystic kidney disease. Ann Intern Med. 2013; 159:382-389. [PubMed: 24042366]

2. Torres VE, Chapman AB, Devuyst O, Gansevoort RT, Grantham JJ, Higashihara E, et al. TEMPO 3:4 Trial Investigators. Tolvaptan in patients with autosomal dominant polycystic kidney disease. N Engl J Med. 2012; 367:2407-2418. [PubMed: 23121377]

3. Levey AS, Stevens LA, Schmid CH, Zhang YL, Castro AF 3rd, Feldman HI, et al. CKD-EPI (Chronic Kidney Disease Epidemiology Collaboration). A new equation to estimate glomerular filtration rate. Ann Intern Med. 2009; 150:604-120. [PubMed: 19414839] 